Proceedings of the 2011 Winter Simulation Conference

S. Jain, R. R. Creasey, J. Himmelspach, K. P. White, and M. Fu, eds.

\title{
TOWARDS SIMULATION OF ORGANIZATIONAL NORMS
}

\author{
Oana Nicolae \\ Department of Informatics \\ Brandenburg University of Technology \\ Walther-Pauer Strasse 2 \\ 03046, Cottbus, Germany
}

\author{
Gerd Wagner \\ Department of Informatics \\ Brandenburg University of Technology \\ Walther-Pauer Strasse 2 \\ 03046, Cottbus, Germany
}

\begin{abstract}
Unlike social norms, which are the unplanned, unexpected result of the interactions among human individuals, organizational norms are stipulated by the organization with the purpose of constraining the behavior of organizational actors in the context of business processes. We propose a simple conceptual model of organizations and organizational norms as an extension of the metamodel of the Agent-Object-Relationship $(A O R)$ simulation language. In our approach an organization is modeled as an institutional agent with organizational units and human actors as subagents that participate in business processes involving other agents, which are possibly affiliated with other organizations. For simplicity, we consider only the most basic form of behavior, which is reactive behavior described in the form of reaction rules, and the most basic types of organizational norms, which are rights and duties defined for organizational positions and roles.
\end{abstract}

\section{INTRODUCTION}

We propose a simple conceptual model of organizations and organizational norms as an extension of the metamodel of the Agent-Object-Relationship (AOR) simulation language AORSL. Organizations are modeled as institutional agents with organizational units and human actors as subagents that participate in business processes involving other agents, which are possibly affiliated with other organizations.

For simplicity, we consider only the most basic form of behavior, which is reactive behavior based on reaction patterns described by reaction rules. In AORSL, these rules can be defined for all kinds of agent types, including organizational roles and organizational positions. Thus, the behavior of a specific agent is defined by merging the behaviors attached to all roles played and all positions held by the agent.

The most basic types of organizational norms are rights and duties defined for organizational positions and roles. They constrain the reactive behavior defined for the positions and roles in the sense that the corresponding reaction rules must comply with these norms.

The remainder of this paper is organized as follows. Section 2 briefly reviews related work in social philosophy, organization theory and Artificial Intelligence. In Section 3 we propose an extension of AORSL by adding the normative concepts of rights and duties. Section 4 describes a test case using the AORS organizational concepts, and the last section discusses conclusions and future works.

\section{RELATED WORK}

Different norm definitions and norm ontologies exist nowadays in the literature. Encyclopedia Britannica defines the concept of norm by relating it to its sociological roots as rule or standard of behavior shared by members of a social group. Social philosophy treats norms as: (1) sentences originated from the area of modal/deontic logic in form of obligations, permissions and prohibitions; or (2) concepts based on some teleological accounts such as: performing some actions, having beliefs and feelings. In Artificial Intelligence 


\section{Nicolae and Wagner}

(AI), mainly in MAS - a branch of AI, the concept of norm is represented as a formal specification of a deontic statement that aims at regulating the life of software agents and the interactions among them. It can take the form of an obligation, a permission or a prohibition, and is often represented with some dialect or extension of deontic logic.

The semantics and the use of the norm concept are also regarded differently by various disciplines such as: political science, anthropology, psychology, economics, legal theory and marketing. (Ott and Ivens 2009) provides a review of norm definitions and norm usages.

(Searle 1995) develops a philosophical theory about social reality, in which he defines:

- regulative rules - rules that stipulate correct behavior when certain social actions are performed in the context of an organization: (1) obligation rules; and (2) permission rules or rights.

- constitutive rules - rules that define the set of constraints which have to be obeyed when the social concepts of an organization are created, modified or dropped.

The formula "x counts as y in c" represents the pattern for constitutive rules which define the social concepts as constitutive elements of some social form, in particular of institutions and organizations. Searle explains: (Searle 1995, I find the formula "x counts as y in c" immensely useful because it gives us a way of articulating the distinction between those functions where the function is performed in virtue of an intrinsic physical feature of the object, and those functions which are performed in virtue of collective recognition of a status.)

In Searle's writings we often find the term deontic powers which he uses to talk about all kind of norms such as: rights, duties, obligations, requirements permissions, authorizations, entitlements, and so on.

(Tuomela 2002) considers the following kinds of norms:

- rule norms, which can be formal when enforced through institutions (like university regulations) or informal, when they concern practices that are not institutionalized (e.g., rules that are established by parents for their children in the context of a family).

- social norms, which involve collective acceptance and mutual expectations (e.g., table manners), therefore they concern a social group.

Organizational norms are similar to what Tuomela calls 'rule norms': formal rules like rights and obligations. We argue that for modeling organizations and their business processes, we may abstract from the cultural layer and its corresponding set of social norms.

(Bottazzi and Ferrario 2009) mention other types of norms:

- normative descriptions - also mentioned in (Santos, Almeida, and Guizzardi 2010) which are somehow equivalent in semantics with Searle's constitutive rules.

- technical norms - they describe the correct procedure to perform certain tasks and they do not have a mandatory aspect.

Norm concepts are also considered in the multi-agent system literature. But often, they are indirectly referred and somehow mixed with the concept of role, usually understood as a set of permissions, as in (Omicini 2001), (Dignum 2004), (Wooldridge, Jennings, and Kinny 1999), (Zambonelli, Jennings, and Wooldridge 2003). A further step in the direction of representing the role concept as a set of permissions and prohibitions, it is done by (Wooldridge, Jennings, and Kinny 2000) and (Cabri, Leonardi, and Zambonelli 2003): they add to the role concept the set of duties. A complex ontology of organizational norms is described by OperA methodology in (Dignum 2004).

(Wagner 2003) introduces the deontic logic of the AOR modeling, as the set of: (1) duties to perform certain actions; (2) the rights to perform certain actions. It further distinguishes between rights as permissions and rights as prohibitions. Moreover, the paper discusses the particular case of normative undetermination which is natural to happen inside of human organizations: (Wagner 2003, it needs not be the case that for 


\section{Nicolae and Wagner}

every agent $\mathrm{i}$, and every action $\alpha$, either $\mathrm{i}$ has the right to do actions of type $\alpha$, or it is prohibited for i to perform actions of type $\alpha$.)

(Grossi, Aldewereld, and Dignum 2006) introduce a new way of categorizing the normative layer inside of electronic institutions by distinguishing the category of regimentations from the other kinds of norms: (1) regimentations have the meanings of prohibitions and they are used in order to avoid serious damages caused by the non-compliance to the specified norms; and (2) other type of norms: duties and permissions.

(Figueiredo and da Silva 2011) describe a norm ontology employed by the language NormML. It considers only the deontic aspects of a normative layer which constraints the behavior of individuals such as: obligations, permissions and prohibitions and does not take into account the rules which constructs the social institution or organizations. (Figueiredo and da Silva 2011) make an interesting remark by associating the deontic constraints not only to individuals and institutions, but also to communicative actions such as: sending/receiving messages and to the state of the system, what they call the environmental context or organizational context. Moreover, NormML considers the definition of sanctions when a norm it is violated e.g punishments. Additionally, the language provides the definition of a special type of sanctions: the rewards received by the entities when a norm it is fulfilled. We do consider the concept of rewards to be meaningful for the definition of the normative layer inside of institutions and organizations.

\section{ADDING ORGANIZATIONAL NORMS TO AORSL}

For the sake of having a self-contained paper, we briefly summarize the organizational concepts already introduced in (Nicolae and Wagner 2011). The AOR simulation language is based on the AOR modeling language proposed in (Wagner 2003). A basic concept of activities has been introduced in (Wagner, Nicolae, and Werner 2009). We discuss in this Section the normative concepts which extend the AOR simulation language.

We have to distinguish between norms that construct an organization in an organizational charter and norms that define behavioral constraints for the subagents of an organization.

\subsubsection{Organizational Charter}

The concept of OrganizationalCharter is responsible for the creation, modification and dropping of the social entities which populate the institutional context. Moreover, the Institutional Charter should be understood as a set of commonly agreed conventions or descriptive norms which are responsible for creating the entire structure of one organization. The Institutional Charter governs the Organization together with its positions and corresponding roles sets. The charter may include ad-hoc norms which are used to prescribe certain behavior to accomplish certain tasks (Searle 1969, one could not perform if these rules did not exist, pp. 33), e.g. one could not:

- $\quad$ name a new staff member of the Chair.

- dismiss one staff member of the Chair.

if the rules of structuring a Faculty's Chair into positions did not exist.

One important characteristic of the institutional charter, one must notice, is the fact that the norms it contains are not susceptible to violations, and therefore they do not attract punishments or rewards, e.g.

- none of the rules of structuring a Faculty's Chair commit the Chair's leader to name a new staff member, nor not to name a new staff member of the Chair.

- none of the rules of structuring a Faculty's Chair commit the Chair's leader to dismiss one staff member, nor not to dismiss one staff member of the Chair. 


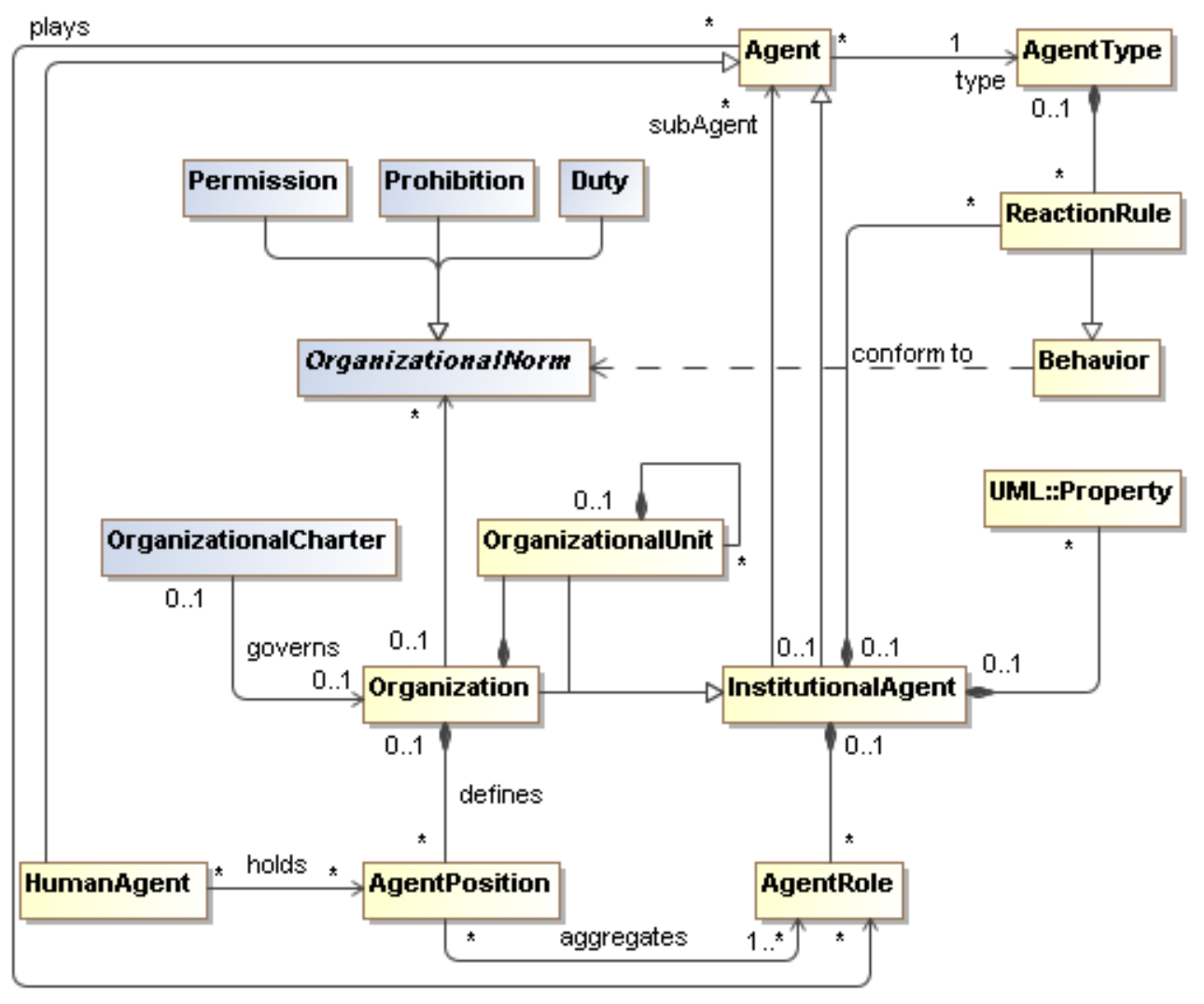

Figure 1: Organizational Concepts - AOR metamodel.

\subsubsection{Organizational Norms}

The concept of OrganizationalNorm is defined at the level of organizations and their subUnits, roles and positions. Organizational norms represent the set of principles and rules which apply constraints on the operational behavior defined by means of ReactionRule(s), which are a particular type of AOR behaviors. We represent these constraints in the UML class diagram by a dependency arrow which shows that the Behavior class conforms to the OrganizationalNorm(s). By using Deontic Logic as a formal basis, one may translate the AOR abstract concept of OrganizationalNorm into: duties (obligations), permissions and prohibitions applied to the reactive behavior of the following AOR organizational agents such as:

- organization and its defined positions and roles.

- human agents which are seen as subagents of institutions and organizations.

The organizational norms are valid during a particular time interval. Therefore, even if the time is not directly a characteristic of the normative layer, but obviously characterizes more complex entities such as complex events, or activities (Wagner, Nicolae, and Werner 2009), when we specify organizational norms we must think that their validity can be influenced by their association with some specified $d u e$ time. Usually, the due time is represented inside of the organizational norms using expressions such as: (1) from begin date until end date; and (2) until end date, e.g. The Erasmus students who want to study at the BTU Cottbus in the Winter Semester 2011/2012 have the duty to enrol at the BTU Students Office until 07.10.2011.

We envision the following categories of organizational norms: 


\section{Nicolae and Wagner}

- Duties - the Duty concept is a subclass of the abstract OrganizationalNorm concept and stipulates constraints on the agents behaviour. The duties and their dependencies with the agent's behavior are expressed in terms of ReactionRule(s) and specified at the level of human agent types, organizations, roles, or positions:

- the duty to react to certain events - for each defined duty, there must be at least one reactive rule which handles each type.

duty ${ }_{1}$ : Any BTU student must pay the Semester Ticket on each Semester.

- Permissions - the Permission concept is a subclass of the abstract OrganizationalNorm concept and describes the allowed agent's behavior:

- the permission to react to certain events - for every defined event which may trigger a reactive rule from the behavior set, there should be specified permissions for the resulting actions, or none of the resulting actions should be prohibited.

As a consequence of accomplishing the duty $y_{1}$, the BTU students have the following permissions, or rights:

permission 1 : Any BTU student has the right to ask for low-cost accommodation on the students campus (Studentenwerk).

permission $:$ Any BTU student has the right of traveling for free in the Brandenburg state by any means of transportation.

- Prohibitions - the Prohibition concept is a subclass of the abstract OrganizationalNorm concept and describes the forbidden agents behavior:

- the prohibition to react to certain events - for every defined event which may trigger a reactive rule from the behavior set, there should be specified prohibitions for the resulting actions, or none of the resulting actions should have specified a permission.

prohibition $_{1}$ : It is prohibited that a BTU student takes an exam for which she is not enrolled before. prohibition $_{2}$ : It is prohibited that a BTU student submits a project assignment after the announced deadline.

As shown below in Figure 2, the abstract OrganizationalNorm concept subsumes under the AOR reactive behavior the following kinds of norms:

- Duties in the sense of Reaction Rules, without possible cases of duties violation due to the agent's reactive behavior.

- Prohibitions in the sense of Reaction Rules, without possible cases of prohibitions violation due to the agent's reactive behavior.

The formalization of the AOR Organizational Norms implies a temporal implication such as:

$p \rightarrow q$, where $p$ and $q$ represent state structures, e.g., properties, conditions and event occurrences. Expressed in the natural language, the temporal implication translates into the following form: whenever $p$, then after some time $q$. Under the AOR simulation language reactive behavior, an equivalent definition would represent the organizational norm, in particular the duty, as: the reaction to a triggered event, by performing some actions after some time. Taking into account the above definition and the reactive nature of the AOR simulation language we represent the duties and the prohibitions using the following pseudo-code:

WHEN Event Type

IF condition

DO AFTER_SOME_TIME Actions

One important characteristic of the organizational norms is the fact that they are conditional: certain conditions must hold for creating the appropriate context for scheduling the actions implied by the organizational norm. The temporal constraint AFTER_SOME_TIME expresses a relative time duration which is justified by the fact that the actions implied by the organizational norm may be scheduled for execution as a result of a reaction rule chain. 
Nicolae and Wagner

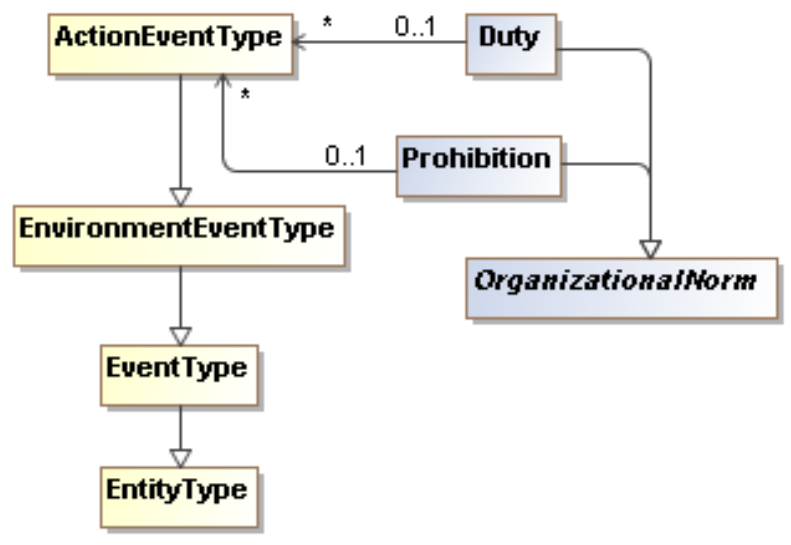

Figure 2: AOR Organizational Norms under Reactive Behavior.

As a consequence of the above explained reactive behavior implemented by the ReactionRules, the agents react to all the events triggering a specified Reaction Rule from the behavior set and they perform all the actions scheduled by the Reaction Rule. In this sense, we argue that the reactive behavior of the agents enforces the execution of all specified norms (duties and prohibitions), so that possible cases of norm violations do not exist.

Moreover, the prohibitions are envisioned as disjunctive with respect to the permissions set and it is obvious that we can model the permissions for events and actions, simply by not mentioning any prohibitions: everything that is not forbidden it is allowed. We choose to explicitly represent the prohibitions and indirectly refer to permissions, because they (prohibitions) can be seen as alarm signals pointing out the negative consequences caused by their hypothetical violation.

\subsection{AOR Organizational Concepts}

\subsubsection{Agent BaseType}

The concept AgentBaseType defines the set of common properties of the agents. Following the approach defined in (Guizzardi and Wagner 2004) and (Guizzardi 2005) the agents must be of a certain AgentBaseType, also called a rigid type in (Guizzardi 2005) which determines the fundamental characteristics of the agents and also provides the identity criterion for its instances, e.g. the Person concept usually represents the baseType for the individuals inside of one institution or organization.

\subsubsection{Agent Role}

The concept AgentRole defines the common characteristics of the roles which exists inside the social system, therefore it must be understood as a concept type. One or many roles are aggregated into AgentPositions. AgentRole(s) represent the functions that are performed by individuals, members of the organization, on behalf of the organization. We borrowed the AgentRole concept's semantics from (Guizzardi and Wagner 2004) and (Guizzardi 2005), where it is defined as an anti-rigid entity: (Guizzardi and Wagner 2004, its instances could possibly also not be instances of it without losing their identity). The AgentRole(s) are defined by the OrganizationalCharter and consequently they are constitutive elements of any social institution. There exist also some dependencies between roles, in the sense that they may be disjunctive (a reviewer of an article can not be an author of the same article) or conjunctive (in order to assume a particular role, one should previously assume another role(s)). Inside of an institution or organization many Agent(s) can play many AgentRole(s). By agents playing roles inside of an institution we understand: (1) 


\section{Nicolae and Wagner}

human agents which populate the institution; and (2) organizational subunits which model the structure of an organization.

\subsubsection{Agent Position}

The concept AgentPosition defines the common characteristics of the agent positions existing inside of the Organization, therefore it must be understood as a concept type. Individuals may assume certain positions within the hierarchy of the organization they belong to. The AgentPosition(s) represent the constitutive elements for the structure of an Organization and they are defined by the OrganizationalCharter. One AgentPosition aggregates at least one AgentRole. When the AgentPosition corresponds to exactly one AgentRole the position equates with that particular role, e.g. AcademicStaff is an example of an agent position type, which is a constitutive element of the university chair. The position type aggregates the role of professor which is the chair's leader and one or many teaching assistant roles, e.g. the position of Faculty's Dean implies a set of duties and tasks that the individual playing this role must accomplish, but also some rights justified by its position status.

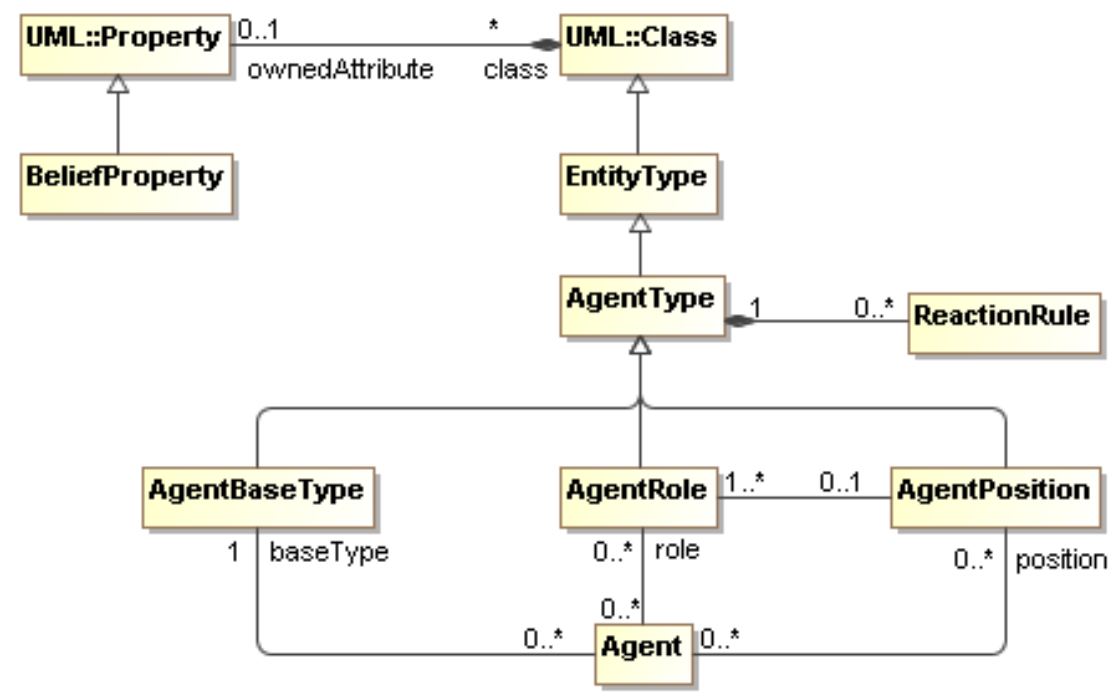

Figure 3: AOR Types metamodel.

\subsubsection{Institutional Agent}

We use the concept of InstitutionalAgent(s), first mentioned in (Wagner 2003), to represent the social institution. The InstitutionalAgent subsumes its members as subAgents, which can be: human agents or organization subUnits (notice the association between the InstitutionalAgent class and the Agent). The InstitutionalAgent is a subclass of the Agent class, therefore one should not considerate it as a type. Notice that we allow to specify supplementary properties and behaviors for the InstitutionalAgent in addition to the default ones specified at the agent and role type level, e.g. the institution of family is represented in the AOR simulation language as an InstitutionalAgent. We follow the approach of (Seumas 2011) and we make distinction between the concept of institution and the concept of organization. The former can subsume a hierarchy of organizations and also can define meta-organizations, as organizations which define other organizations. 


\section{Nicolae and Wagner}

\subsubsection{Organization}

The Organization concept, see 1, it is defined in our metamodel as a subclass of the InstitutionalAgent and its first functionality is to define the OrganizationalNorm(s) that govern and regulate the operational behavior of the organization and its members. The structure of the Organization is entirely defined and constrained by the OrganizationalCharter.The Organization concept is enhanced with the following characteristics: (1) the possibility to have as constitutive elements zero or many AgentPosition(s) which aggregate roles; and (2) the possibility to define a hierarchy of OrganizationalUnits inside of the organization by using the one-to-many aggregate association of the OrganizationalUnit class with itself, e.g. the University is an example of organization, it defines a hierarchy of positions which aggregate roles and displays a hierarchy of departments as organizational subUnits.

\subsection{AOR Core Concepts}

\subsubsection{Agent}

We distinguish between:

- organizations represented as institutional agents, such as universities;

- organizational units such as the faculties of a university;

- people represented as human agents;

- other kinds of artificial agents included by the type Agent such as: computer programs.

Each agent must be an instance of: (1) an unique agent base type which provides to its instances the existential criterion; (2) zero or many agent position(s); and (3) zero or many agent role(s) (see Figure 3). There must be a dependency relationship between the AgentBaseType and the other AgentRole(s) in the sense that an individual can instantiate only the role types which are in the dependency relationship with their base type, e.g. the roles types Student and Professor are in a dependency relationship with the base type Person, but not with another possible base type, such as Child.

\subsubsection{Agent Type}

The AgentType class defines the common set of properties (UML::Property class) and behavior expressed by means of ReactionRule(s) for all agent type's instances. The concept inherits the EntityType class, meaning that in our model the agents are considered to be entities. The reactive rules define the default normative behavior: the duty to react to a certain set of events by triggering the reaction rules which model the behavior of the base types and of the agent role types instances. AOR simulation language offers support for agent beliefs which are represented in the language also as properties of the AgentType concept.

The beliefs allow agents to preserve information (possible false) about themselves, about other entities or about events that may occur. AOR simulation language offers support for two types of agent beliefs explained in one of the following sub-sections:

- $\quad$ self beliefs - are beliefs of an agent about itself. Such beliefs may refer to physical or non physical properties of the agent.

- belief entities - are beliefs of an agent about other agents, objects or events.

We consider it to be necessary to mention the existence of agent's beliefs and therefore to consider the individuals as cognitive agents, because the idea of a norm is an inherently a social idea that needs to be embodied in ones actions, beliefs and feelings (Searle 1995). 
Nicolae and Wagner

\subsubsection{Reaction Rules}

ReactionRule(s) are the main AOR simulation language constructs which model the reactive behavior of the agents. In the literature they are also called ECA(Event-Condition-Action) rules. They define the agent's reactive behavior in response to perception events and internal time events. Reaction rules are aggregated by the AgentType concept, therefore the agent base type, AgentPosition and AgentRole concepts can define the reactive behavior of their instances. Reaction rules can also be specified at the level of the InstitutionalAgent. This means that one can specify additional behavior at the level of institutions and organizations which are considered individual agents of a certain type. Reaction rules are also the subject of behavior constraints exercised by the normative layer of the institution in the form of norms which regulate the behavior of institutions and of their members, e.g. Organizational Norms.

\section{UNIVERSITY - A NORMATIVE ORGANIZATION CASE STUDY}

We choose to model an academic environment and the normative, organizational concept of University is the first choice to reach. Brandenburg University of Technology Cottbus (BTU Cottbus) is an example which can successfully instantiate such an organization structure and its normative behavior. Due to the complex structure of an University, we choose to only briefly represent the University's inner structure including the official positions and their aggregated roles in terms of concepts defined by the extension of the AOR simulation language together with the normative layer which governs the organization (see Figure 4). The UML diagram comprises:

- the AOR meta-types expressed as stereotypes of the concept types classes, e.g., organization, organizational unit, agent position, agent role, human agent.

- the concept types such as: University, Faculty, Chair, Person, Chair Holder, Professor.

- the individuals - instances of the organizational concept types such as: BTU Cottbus.

Our use case abstracts away from some obvious elements which are constitutive for an University, e.g. organization subunits such as: Senate, Presidential Body, position types such as: President of the University etc. with the purpose of simplifying the resulting UML class diagram. We focus only on representing the Faculties as the main organization subunits of the University, and the Chairs as the main organizational subunits of the Faculty concept. Our purpose is to show the organizational structure in terms of organizational units, such as a Faculty Chair, and the organizational subunits in terms of agent positions and their aggregated roles. We show how the OrganizationalCharter actually defines and regulates the structure of the entire organization, and we model the operational behavior of the University by defining appropriate OrganizationalNorms.

\subsection{Organizational Charter}

Notice that the OrganizationalCharter concept from the UML class diagram defines the entire structure of the University, its Faculties and Chairs in terms of: organization subunits, agent position types and corresponding, aggregated roles. The structure of a Faculty's Chair inside of BTU Cottbus should comprise the following positions: one Chair Holder (the professor), one secretary of the Chair, one optional technical assistant and at least one teaching assistant. By defining the positions as constitutive elements of a Faculty's Chair, one should notice their cardinality. This means that indeed the agent position is a concept type which can be further instantiated by one or more individuals who fill the positions, e.g. the teaching assistant position type is instantiated by at least one actual teaching position which, at its turn, is filled in by a person. One particular case is the teaching assistant position type which can define two distinct positions inside of a Faculty's Chair. 


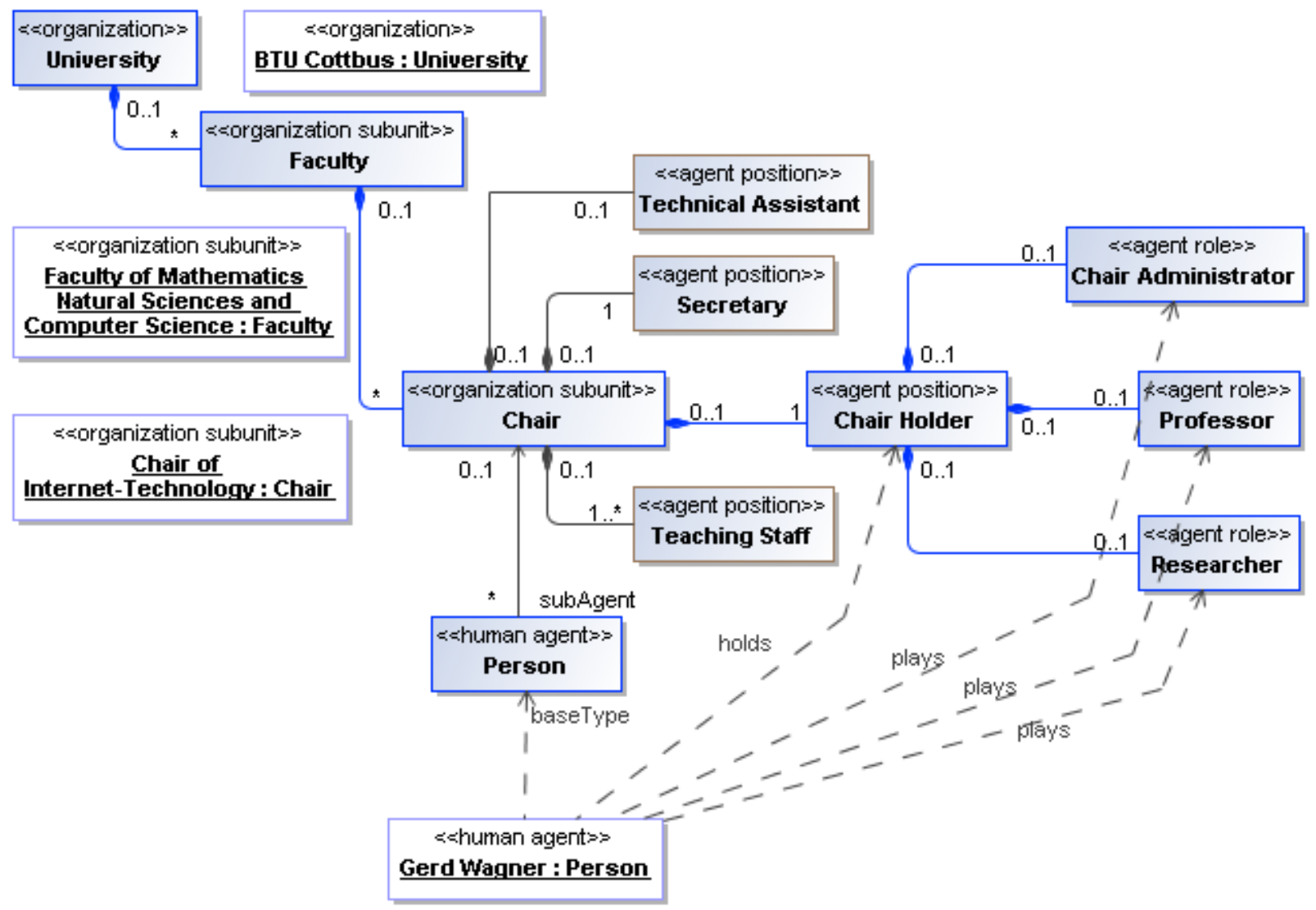

Figure 4: BTU Cottbus - Normative Organization.

\subsection{Organizational Norms}

If we take, for example, the case of Chair Holder as an agent position, from the Figure 4 we can notice the aggregated agent roles: Professor, Researcher and Chair's Administrator. Each of the roles implies some functionality and operative behavior. Therefore the University defines for each Chair Holder the following duties expressed in natural language:

duty $y_{1}$ : Each Chair Holder has the duty to organize study courses and examinations.

duty $y_{2}$ Each Chair Holder has the duty to coordinate the research within the Chair.

Each of the duties described above corresponds to one or more action(s), e.g., to solve, to organize, to coordinate which are to be performed inside of the organizational context: the faculty's Chair. One may notice the general character of the duties which are described at the level of a position type, e.g. Chair Holder, within the general context of a organization subunit type, e.g. the Chair.

As described in Figure 4, the individual Gerd Wagner has the following characteristics:

- is an instance of baseType Person class, which inherits the HumanAgent AOR meta-type.

- $\quad$ is a member of the organizational unit Chair.

- holds the positions of Chair Holder within the Chair organizational subunit.

- plays the following agent roles aggregated by the chair Holder position: Chair Administrator, Professor and Researcher. 


\section{Nicolae and Wagner}

Prohibitions, same as duties, can be applied: (1) at the level of a position type or of a role type in the context of a organization type; and (2) more precisely, to one particular person in a particular context:

prohibition $_{1}$ : It is forbidden for every BTU employee to refuse to accomplish his/her work duties without a professional justification.

prohibition $_{2}$ : It is forbidden for every BTU PhD student to not finish his/her doctorate thesis within six years.

\section{CONCLUSION AND FUTURE WORKS}

The aim of the paper is to describe an abstract syntax for some normative behavior modeling constructs by extending the Agent-Object-Relationship (AOR) simulation language. We propose the following categories of organizational norms: the organizational charter which constructs the organizational structure and the organizational norms which define the prohibitions and the duties attached to positions, roles and human agents, and which are applied in the context of an institution or of an organization. In this paper we informally discussed the organizational charter and norms under the reactive behavior of the agents.

Our future works focus on the formalization of the AOR organizational norms, and on the investigation of the AOR organizational norms under the proactive behavior of the agents. In a proactive context, the agents are not enforced to react to the events and, in consequence, not all the Reaction Rules from the behavioral set may be triggered and, in consequence, not all the scheduled actions may be performed. On the other side, the agents which have a proactive behavior may choose to perform actions which are prohibited. This results in norm violation which attracts legal sanctions or punishments. While the duties and the prohibitions may attract sanctions in case of their disobedience, the Permissions due to their semantics as rights, are not subject of legal punishments or sanctions.

The abstract OrganizationalNorm concept would subsume an enhanced ontology of norms comprising: duties, prohibitions, permissions and sanctions as consequences of duties and prohibitions violations. Notice that not all prohibitions and not all duties may face the legal aspect of punishment. For example, the prohibition $_{1}$ defined in Section 3.0.2 Organizational Norms may be violated, and no legal sanctions, such as expulsion, are applied. But, there is a loss in the fact that the student is not able to give the exam and obtain a grade. In this case, the sanction is not interpreted as a legal punishment, but only as a personal loss, a weak case of punishment.

\section{REFERENCES}

Bottazzi, E., and R. Ferrario. 2009. "Preliminaries to a DOLCE ontology of organisations". Int. Journal Business Process Integration and Management 4:225-238.

Cabri, G., L. Leonardi, and F. Zambonelli. 2003. "BRAIN: A Framework for Flexible Role-Based Interactions in Multiagent Systems". In CoopIS/DOA/ODBASE, edited by R. Meersman, Z. Tari, and D. C. Schmidt, $145-161$.

Dignum, V. 2004. A Model for Organizational Interaction: Based on Agents, Founded in Logic. Ph.D. thesis, Institute for Information and Computing Sciences, University of Utrecht, The Netherlands, Utrecht, The Netherlands. Available via http://igitur-archive.library.uu.nl/dissertations/2003-1218-115420/full. pdf [accessed June 07, 2011].

Figueiredo, K., and V. T. da Silva. 2011. "Norm-ML: A Modeling Language to Model Norms". In ICAART (2), edited by J. Filipe and A. L. N. Fred, 232-237. Rome, Italy: SciTePress.

Grossi, D., H. Aldewereld, and F. Dignum. 2006. "Ubi Lex Ibi Poena: Designing Norm Enforcement in E-Institutions". In Lecture Notes in Agent Systems II, Coordination, Organizations, Institutions and Norms (COIN), vol 4386, edited by P. Noriega, J. Vazquez-Salceda, G. Boella, O. Boissier, V. Dignum, N. Fornara, and E. Matson, 101-114. Hakodate, Japan. 
Guizzardi, G. 2005. Ontological Foundations for Structural Conceptual Models. Ph.D. thesis, Centre for Telematics and Information Technology, University of Twente, The Netherlands, Twente, The Netherlands. Available via http://doc.utwente.n1/50826/1/thesis_Guizzardi.pdf [accessed April 1, 2011].

Guizzardi, G., and G. Wagner. 2004. On a Unified Foundational Ontology and Some Applications of it in Business Modeling, Ontologies and Business System Analysis. IDEA Group.

Nicolae, O., and G. Wagner. 2011. "Modeling and Simulationg Organisations". In Enterprise and Organizational Modeling and Simulation, Vol. 88, edited by T. E. J. Barjis and A. Gupta, Lecture Notes in Business Information Processing, 45-62. London, UK: LNBIP.

Omicini, A. 2001. "SODA: Societies and infrastructures in the analysis and design of agent-based systems". In Lecture Notes in Computer Science, Agent-Oriented Software Engineering (AOSE), vol 1957, edited by P. Ciancarini and M. Wooldridge, 185-193. Limerick, Ireland.

Ott, C., and B. Ivens. 2009. "Revisiting the norm concept in relational governance". Industrial Marketing Management 38:577-583.

Santos, P. S. J., A. J. Almeida, and G. Guizzardi. 2010. "An Ontology-Based Semantic Foundation for Organizational Structure Modeling in the ARIS Method". Enterprise Distributed Object Computing Conference Workshops, IEEE International 0:272-282.

Searle, J. R. 1969. Speech Acts. New York: Cambridge University Press.

Searle, J. R. 1995. The construction of Social Reality. The Free Press.

Seumas, M. 2011. "Social Institutions". The Stanford Encyclopedia of Philosophy. Available via http: //plato.stanford.edu/archives/spr2011/entries/social-institutions/ [accessed August 10, 2011].

Tuomela, R. 2002. The Philosophy of Social Practices: A Collective Acceptance View. Cambridge University Press.

Wagner, G. 2003. "The Agent-Object-Relationship Metamodel: Towards a unified view of state and behavior". Information Systems 5:475-504.

Wagner, G., O. Nicolae, and J. Werner. 2009, December. "Extending Discrete Event Simulation by adding an Activity concept for Business Process Modeling and Simulation". In Proceedings of the 2009 Winter Simulation Conference, edited by M. D. Rossetti, R. R. Hill, B. Johansson, A. Dunkin, and R. G. Ingalls, 2951-2962. Piscataway, New Jersey: Institute of Electrical and Electronics Engineers, Inc.

Wooldridge, M. J., N. R. Jennings, and D. A. Kinny. 1999. "A methodology for agent-oriented analysis and design". In Proceedings of the 3rd International Conference on Autonomous Agents, edited by J. P. M. O. Etzioni and J. Bradshaw, 285-312.

Wooldridge, M. J., N. R. Jennings, and D. A. Kinny. 2000. "The Gaia methodology for agent-oriented analysis and design". Journal of Autonomous Agents and Multi-Agent Systems 3:285-312.

Zambonelli, F., N. R. Jennings, and M. Wooldridge. 2003. "Developing multiagent systems: The GAIA methodology". Transactions on Software Engineering and Methodology 3:317-370.

\section{AUTHOR BIOGRAPHIES}

GERD WAGNER is Professor of Internet Technology within the Department of Informatics, Brandenburg University of Technology. His research interests include agent-oriented modeling and agent-based simulation, foundational ontologies, (business) rule technologies and the Semantic Web. In recent years, he has been focusing his research on the development of an agent-based discrete event simulation framework, called AOR Simulation. He can be reached at http://www.informatik.tu-cottbus.de/ $\sim$ gwagner/. His e-mail address is G.Wagner@tu-cottbus.de.

OANA NICOLAE is currently a PhD student within the Department of Informatics, Brandenburg University of Technology Cottbus, Germany. Her research interests comprise: Modeling and Simulation of Business Processes inside of Organizations, Orchestrations and Choreographies of Business Processes, Conceptual Modeling with UML / BPMN / BPDM. She can be reached at http://oxygen.informatik.tu-cottbus.de/ $\sim$ nicolae/. Her e-mail address is Oana.Nicolae@tu-cottbus.de. 